I 19.2:EL2/3/991

U.S. Geological Survey

in

\title{
Elevations and Distances in the United States
}

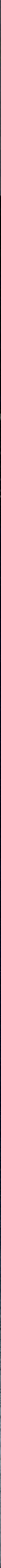





\section{NOT FOR CIRCULATION}

\section{Elevations and Distances}

\section{in the United States}

The information in this booklet was compiled to answer inquiries received by the U.S. Geological Survey from students; teachers; writers; editors; publishers of encyclopedias, almanacs, and other reference books; and people in many other fields of work.

The elevations of features and distances between points in the United States were determined from surveys and topographic maps of the U.S. Geological Survey or obtained from other sources. In most cases, the elevations were determined from surveys and from 1:24,000- and 1:25,000-scale, 7.5-minute topographic quadrangle maps. In Alaska, information was taken from 1:63,360-scale, 15-minute topographic quadrangle maps. In a few cases, data were obtained from older, 1:62,500-scale, 15-minute maps; these maps are being replaced with larger-scale 7.5-minute coverage. Further information about U.S. Geological Survey products can be obtained from: U.S. Geological Survey, Earth Science Information Center, 507 National Center, Reston, VA 22092 or phone 703-860-6045.

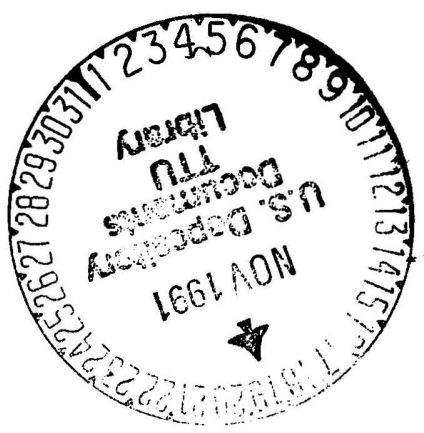




\section{Elevations of the $\mathbf{5 0}$ Largest Cities (by population, 1980 Census)}

\begin{tabular}{|c|c|c|c|c|}
\hline City & State & Rank & $\begin{array}{l}\text { High } \\
\text { feet }\end{array}$ & $\begin{array}{l}\text { Low } \\
\text { feet }\end{array}$ \\
\hline New York & New York & 1 & 410 & Sea Level \\
\hline Chicago & Illinois & 2 & 673 & 1579 \\
\hline Los Angeles & California & 3 & 5,074 & Sea Level \\
\hline Philadelphia & Pennsylvania & 4 & 441 & Sea Level \\
\hline Houston & Texas & 5 & 83 & Sea Level \\
\hline Detroit & Michigan & 6 & 672 & 1575 \\
\hline Dallas & Texas & 7 & 686 & 390 \\
\hline San Diego & California & 8 & 823 & Sea Level \\
\hline Phoenix & Arizona & 9 & 1,160 & 1,058 \\
\hline Baltimore & Maryland & 10 & 489 & Sea Level \\
\hline San Antonio & Texas & 11 & 1,000 & 505 \\
\hline Indianapolis & Indiana & 12 & 845 & 664 \\
\hline San Francisco & California & 13 & 934 & Sea Level \\
\hline Memphis & Tennessee & 14 & 335 & 195 \\
\hline Washington & D.C. & 15 & 410 & 1 \\
\hline Milwaukee & Wisconsin & 16 & 799 & 1579 \\
\hline San Jose & California & 17 & 2,125 & Sea Level \\
\hline Cleveland & Ohio & 18 & 1,050 & 1571 \\
\hline Columbus & Ohio & 19 & 893 & 685 \\
\hline Boston & Massachusetts & 20 & 330 & Sea Level \\
\hline New Orleans & Louisiana & 21 & 25 & -8 \\
\hline Jacksonville & Florida & 22 & 40 & Sea Level \\
\hline Seattle & Washington & 23 & 520 & Sea Level \\
\hline Denver & Colorado & 24 & 5,470 & 5,130 \\
\hline Nashville-Davidson County & Tennessee & 25 & 1,160 & 385 \\
\hline St. Louis & Missouri & 26 & 614 & 385 \\
\hline Kansas City & Missouri & 27 & 1,020 & 720 \\
\hline El Paso & Texas & 28 & 4,080 & 3,695 \\
\hline Atlanta & Georgia & 29 & 1,086 & 738 \\
\hline Pittsburgh & Pennsylvania & 30 & 1,370 & 710 \\
\hline Oklahoma City & Oklahoma & 31 & 1,334 & 1,050 \\
\hline Cincinnati & Ohio & 32 & 960 & 433 \\
\hline Fort Worth & Texas & 33 & 766 & 513 \\
\hline Minneapolis & Minnesota & 34 & 980 & 687 \\
\hline Portland & Oregon & 35 & 1,073 & Sea Level \\
\hline Honolulu & Hawaii & 36 & 4,020 & Sea Level \\
\hline Long Beach & California & 37 & 360 & -7 \\
\hline Tulsa & Oklahoma & 38 & 810 & 620 \\
\hline Buffalo & New York & 39 & 699 & 1571 \\
\hline Toledo & Ohio & 40 & 627 & 1571 \\
\hline Miami & Florida & 41 & 30 & Sea Level \\
\hline
\end{tabular}




\begin{tabular}{llrrr} 
City & State & Rank & \multicolumn{1}{c}{$\begin{array}{c}\text { High } \\
\text { feet }\end{array}$} & \multicolumn{1}{c}{$\begin{array}{r}\text { Low } \\
\text { feet }\end{array}$} \\
\hline Austin & Texas & 42 & 1,080 & 406 \\
\hline Oakland & California & 43 & 1,754 & Sea Level \\
\hline Albuquerque & New Mexico & 44 & 6,120 & 4,946 \\
\hline Tucson & Arizona & 45 & 2,950 & 2,250 \\
\hline Newark & New Jersey & 46 & 278 & Sea Level \\
\hline Charlotte & North Carolina & 47 & 784 & 519 \\
\hline Omaha & Nebraska & 48 & 1,272 & 960 \\
\hline Louisville & Kentucky & 49 & 761 & 382 \\
\hline Birmingham & Alabama & 50 & 1,060 & 496 \\
\hline
\end{tabular}

1Water level, International Great Lakes Datum.

\section{Highest and Lowest Elevations}

\begin{tabular}{|c|c|c|c|c|c|c|}
\hline $\begin{array}{l}\text { State or } \\
\text { possession }\end{array}$ & $\begin{array}{l}\text { Highest } \\
\text { point }\end{array}$ & $\begin{array}{l}\text { County or } \\
\text { subdivision }\end{array}$ & $\begin{array}{l}\text { Elev. } \\
\text { feet }\end{array}$ & Lowest point & $\begin{array}{l}\text { County or } \\
\text { subdivision }\end{array}$ & $\begin{array}{l}\text { Elev. } \\
\text { feet }\end{array}$ \\
\hline Alabama & Cheaha Mt. & Cleburne & 2,405 & Gulf of Mexico & - & Sea level \\
\hline Alaska & Mt. McKinley & & 20,320 & Pacific Ocean & - & Sea level \\
\hline Arizona & $\begin{array}{l}\text { Humphreys } \\
\text { Peak }\end{array}$ & Coconino & 12,633 & Colorado River & Yuma & 70 \\
\hline Arkansas & Magazine Mt. & Logan & 2,753 & Ouachita River & Ashley-Union & 55 \\
\hline California & Mt. Whitney & $\begin{array}{l}\text { Inyo- } \\
\text { Tulare }\end{array}$ & 114,494 & Death Valley & Inyo & -282 \\
\hline Colorado & Mt. Elbert & Lake & 14,433 & Arkansas River & Prowers & 3,350 \\
\hline Connecticut & $\begin{array}{l}\text { Mt. Frissell } \\
\text { on south } \\
\text { slope at State } \\
\text { line }\end{array}$ & Litchfield & 2,380 & Long Island Sound & - & Sea level \\
\hline Delaware & $\begin{array}{l}\text { On Ebright } \\
\text { Road at } \\
\text { Delaware- } \\
\text { Pennsylvania } \\
\text { state line }\end{array}$ & New Castle & 1448 & Atlantic Ocean & - & Sea level \\
\hline $\begin{array}{l}\text { District of } \\
\text { Columbia }\end{array}$ & $\begin{array}{l}\text { Tenleytown } \\
\text { at Reno } \\
\text { Reservoir }\end{array}$ & - & 410 & Potomac River & - & 1 \\
\hline Florida & $\begin{array}{l}\text { Sec.30, } \\
\text { T.6N., R.20W. }\end{array}$ & Walton & 345 & Atlantic Ocean & - & Sea level \\
\hline Georgia & $\begin{array}{l}\text { Brasstown } \\
\text { Bald }\end{array}$ & $\begin{array}{l}\text { Towns- } \\
\text { Union }\end{array}$ & 4,784 & Atlantic Ocean & - & Sea level \\
\hline Guam & Mt. Lamlam & $\begin{array}{l}\text { Agat } \\
\text { District }\end{array}$ & 1,332 & Pacific Ocean & - & Sea level \\
\hline Hawaii & $\begin{array}{l}\text { Pu'u Wekiu, } \\
\text { Mauna Kea }\end{array}$ & Hawaii & 13,796 & Pacific Ocean & - & Sea level \\
\hline Idaho & Borah Peak & Custer & 12,662 & Snake River & Nez Perce & 710 \\
\hline Illinois & $\begin{array}{l}\text { Charles } \\
\text { Mound }\end{array}$ & $\begin{array}{l}\text { Jo } \\
\text { Daviess }\end{array}$ & 1,235 & Mississippi River & Alexander & 2279 \\
\hline Indiana & $\begin{array}{l}\text { Franklin } \\
\text { Township }\end{array}$ & Wayne & 1,257 & Ohio River & Posey & 2320 \\
\hline
\end{tabular}




\begin{tabular}{|c|c|c|c|c|c|c|}
\hline $\begin{array}{l}\text { State or } \\
\text { possession }\end{array}$ & $\begin{array}{l}\text { Highest } \\
\text { point }\end{array}$ & $\begin{array}{l}\text { County or } \\
\text { subdivision }\end{array}$ & $\begin{array}{l}\text { Elev. } \\
\text { feet }\end{array}$ & Lowest point & $\begin{array}{l}\text { County or } \\
\text { subdivision }\end{array}$ & $\begin{array}{l}\text { Elev. } \\
\text { feet }\end{array}$ \\
\hline lowa & $\begin{array}{l}\text { Sec. } 29, \\
\text { T.100N., R.41W }\end{array}$ & Osceola & 1,670 & Mississippi River & Lee & 480 \\
\hline Kansas & Mt. Sunflower & Wallace & 34,039 & Verdigris River & Montgomery & 679 \\
\hline Kentucky & Black Mt. & Harlan & 4,139 & Mississippi River & Fulton & 2257 \\
\hline Louisiana & Driskill Mt. & Bienville & 535 & New Orleans & Orleans & -8 \\
\hline Maine & Mt. Katahdin & Piscataquis & 5,267 & Atlantic Ocean & - & Sea level \\
\hline Maryland & Backbone Mt. & Garrett & 3,360 & Atlantic Ocean & - & Sea level \\
\hline $\begin{array}{l}\text { Massachu- } \\
\text { setts }\end{array}$ & Mt. Greylock & Berkshire & 3,487 & Atlantic Ocean & - & Sea level \\
\hline Michigan & Mt. Arvon & Baraga & 1,979 & Lake Erie & - & 2571 \\
\hline Minnesota & Eagle Mt. & Cook & 2,301 & Lake Superior & - & 2600 \\
\hline Mississippi & Woodall Mt. & Tishomingo & 806 & Gulf of Mexico & - & Sea level \\
\hline Missouri & Taum Sauk Mt. & Iron & 1,772 & St. Francis River & Dunklin & 2230 \\
\hline Montana & Granite Peak & Park & 12,799 & Kootenai River & Lincoln & $\overline{1,800}$ \\
\hline Nebraska & $\begin{array}{l}\text { Johnson } \\
\text { Township }\end{array}$ & Kimball & 5,424 & Missouri River & Richardson & 840 \\
\hline Nevada & Boundary Peak & Esmeralda & 13,140 & Colorado River & Clark & 479 \\
\hline $\begin{array}{l}\text { New } \\
\text { Hampshire }\end{array}$ & Mt. Washington & Coos & 6,288 & Atlantic Ocean & - & Sea level \\
\hline New Jersey & High Point & Sussex & 31,803 & Atlantic Ocean & - & Sea level \\
\hline New Mexico & Wheeler Peak & Taos & 13,161 & $\begin{array}{l}\text { Red Bluff } \\
\text { Reservoir }\end{array}$ & Eddy & 2,842 \\
\hline New York & Mt. Marcy & Essex & 5,344 & Atlantic Ocean & - & Sea level \\
\hline $\begin{array}{l}\text { North } \\
\text { Carolina }\end{array}$ & Mt. Mitchell & Yancey & 6,684 & Atlantic Ocean & - & Sea level \\
\hline $\begin{array}{l}\text { North } \\
\text { Dakota }\end{array}$ & White Butte & Slope & 3,506 & Red River & Pembina & 750 \\
\hline Ohio & Campbell Hill & Logan & 1,549 & Ohio River & Hamilton & 2455 \\
\hline Oklahoma & Black Mesa & Cimarron & 4,973 & Little River & McCurtain & 289 \\
\hline Oregon & Mt. Hood & $\begin{array}{l}\text { Clackamas- } \\
\text { Hood River }\end{array}$ & 11,239 & Pacific Ocean & - & Sea level \\
\hline $\begin{array}{l}\text { Pennsyl- } \\
\text { vania }\end{array}$ & Mt. Davis & Somerset & 3,213 & Delaware River & Delaware & Sea level \\
\hline Puerto Rico & $\begin{array}{l}\text { Cerro de } \\
\text { Punta }\end{array}$ & $\begin{array}{l}\text { Ponce } \\
\text { District }\end{array}$ & 4,390 & Atlantic Ocean & - & Sea level \\
\hline $\begin{array}{l}\text { Rhode } \\
\text { Island }\end{array}$ & Jerimoth Hill & Providence & 812 & Atlantic Ocean & - & Sea level \\
\hline Samoa & Lata Mt. & Tau Island & 3,160 & Pacific Ocean & - & Sea level \\
\hline $\begin{array}{l}\text { South } \\
\text { Carolina }\end{array}$ & Sassafras Mt. & Pickens & 3,560 & Atlantic Ocean & - & Sea level \\
\hline $\begin{array}{l}\text { South } \\
\text { Dakota }\end{array}$ & Harney Peak & Pennington & 7,242 & Big Stone Lake & Roberts & 966 \\
\hline Tennessee & $\begin{array}{l}\text { Clingmans } \\
\text { Dome }\end{array}$ & Sevier & 6,643 & Mississippi River & Shelby & 2178 \\
\hline Texas & $\begin{array}{l}\text { Guadalupe } \\
\text { Peak }\end{array}$ & Culberson & 8,749 & Gulf of Mexico & - & Sea level \\
\hline Utah & Kings Peak & Duchesne & 13,528 & Beaverdam Wash & Washington & 2,000 \\
\hline Vermont & Mt. Mansfield & Lamoille & 4,393 & Lake Champlain & - & 95 \\
\hline
\end{tabular}




\begin{tabular}{|c|c|c|c|c|c|c|}
\hline $\begin{array}{l}\text { State or } \\
\text { possession }\end{array}$ & $\begin{array}{l}\text { Highest } \\
\text { point }\end{array}$ & $\begin{array}{l}\text { County or } \\
\text { subdivision }\end{array}$ & $\begin{array}{l}\text { Elev. } \\
\text { feet }\end{array}$ & Lowest point & $\begin{array}{l}\text { County or } \\
\text { subdivision }\end{array}$ & $\begin{array}{l}\text { Elev. } \\
\text { feet }\end{array}$ \\
\hline Virginia & Mt. Rogers & $\begin{array}{l}\text { Grayson- } \\
\text { Smyth }\end{array}$ & 5,729 & Atlantic Ocean & - & Sea level \\
\hline $\begin{array}{l}\text { Virgin } \\
\text { Islands }\end{array}$ & Crown Mt. & $\begin{array}{l}\text { St. Thomas } \\
\text { Island }\end{array}$ & 1,556 & Atlantic Ocean & - & Sea level \\
\hline Washington & $\begin{array}{l}\text { Mt. Rainier } \\
\text { West }\end{array}$ & Pierce & 14,410 & Pacific Ocean & - & Sea level \\
\hline $\begin{array}{l}\text { West } \\
\text { Virginia }\end{array}$ & Spruce Knob & Pendleton & 4,861 & Potomac River & Jefferson & 240 \\
\hline Wisconsin & Timms Hill & Price & 1,951 & Lake Michigan & - & 2579 \\
\hline Wyoming & Gannett Peak & Fremont & 13,804 & $\begin{array}{l}\text { Belle Fourche } \\
\text { River }\end{array}$ & Crook & 3,099 \\
\hline $\begin{array}{l}\text { United } \\
\text { States }\end{array}$ & $\begin{array}{l}\text { Mt. McKinley, } \\
\text { AK }\end{array}$ & & 20,320 & Death Valley & Inyo, CA & -282 \\
\hline
\end{tabular}

1National Geodetic Survey. ${ }^{2}$ Corps of Engineers. ${ }^{3}$ State Surveys.

\section{Elevations of Named Summits Over 14,000 Feet Above Sea Level}

\begin{tabular}{lllll}
\hline \multicolumn{1}{c}{ Summit } & State & Quadrangle & Date & $\begin{array}{c}\text { Elevation } \\
\text { feet }\end{array}$ \\
\hline Mt. McKinley & AK & Mt. McKinley A-3 & 1954 & 20,320 \\
\hline Mt. St. Elias & AK & Mt. St. Elias B-8 & 1985 & 18,008 \\
\hline Mt. Foraker & AK & Talkeetna D-3 & 1958 & 17,400 \\
\hline Mt. Bona & AK & McCarthy B-2 & 1959 & 16,500 \\
\hline Mt. Blackburn & AK & McCarthy C-7 & 1959 & 16,390 \\
\hline Mt. Sanford & AK & Gulkana A-1 & 1959 & 16,237 \\
\hline Mt. Vancouver & AK & Mt. St. Elias A-2 & 1985 & 15,979 \\
\hline South Buttress & AK & Mt. McKinley A-2 & 1954 & 15,885 \\
\hline Mt. Churchill & AK & McCarthy B-2 & 1959 & 15,638 \\
\hline Mt. Fairweather & AK & Mt. Fairweather D-5 & 1961 & 15,300 \\
\hline Mt. Hubbard & AK & Mt. St. Elias B-3 & 1985 & 14,950 \\
\hline Mt. Bear & AK & McCarthy B-1 & 1959 & 14,831 \\
\hline East Buttress & AK & Mt. McKinley A-2 & 1954 & 14,730 \\
\hline Mt. Hunter & AK & Talkeetna D-3 & 1958 & 14,573 \\
\hline Browne Tower & AK & Mt. McKinley A-2 & 1954 & 14,530 \\
\hline Mt. Alverstone & AK & Mt. St. Elias B-3 & 1985 & 14,500 \\
\hline Mt. Whitney & CA & Mt. Whitney & 1985 & 114,494 \\
\hline University Peak & AK & McCarthy B-3 & 1959 & 14,470 \\
\hline Mt. Elbert & CO & Mt. Elbert & 1967 & 114,433 \\
\hline Mt. Massive & CO & Mt. Massive & 1967 & 14,421 \\
\hline Mt. Harvard & CO & Mt. Harvard & 1963 & 14,420 \\
\hline Mt. Rainier & WA & Mt. Rainier West & 1982 & 14,410 \\
\hline Mt. Williamson & CA & Mt. Williamson & 1971 & 14,370 \\
\hline La Plata Peak & CO & Mt. Elbert & 1984 & 14,361 \\
\hline Blanca Peak & CO & Blanca Peak & 1967 & 14,309 \\
\hline Uncompahgre Peak & CO & Uncompahgre Peak & 1967 \\
\hline & & & 1967 & \\
\hline
\end{tabular}




\begin{tabular}{|c|c|c|c|c|}
\hline Summit & State & Quadrangle $^{2}$ & Date & $\begin{array}{c}\text { Elevation } \\
\text { feet }\end{array}$ \\
\hline Crestone Peak & $\mathrm{CO}$ & Crestone Peak & 1967 & 14,294 \\
\hline Mt. Lincoln & $\mathrm{CO}$ & Alma & 1970 & 14,286 \\
\hline Grays Peak & $\mathrm{CO}$ & Grays Peak & 1958 & 14,270 \\
\hline Mt. Antero & $\mathrm{CO}$ & Mt. Antero & 1983 & 14,269 \\
\hline Torreys Peak & $\mathrm{CO}$ & Grays Peak & 1958 & 14,267 \\
\hline Castle Peak & $\mathrm{CO}$ & Hayden Peak & 1960 & 14,265 \\
\hline Quandary Peak & $\mathrm{CO}$ & Breckenridge & 1970 & 14,265 \\
\hline Mt. Evans & $\mathrm{CO}$ & Mt. Evans & 1957 & 14,264 \\
\hline Longs Peak & $\mathrm{CO}$ & Longs Peak & 1961 & 14,255 \\
\hline Mt. Wilson & $\mathrm{CO}$ & Mt. Wilson & 1953 & 14,246 \\
\hline White Mt. Peak & $\mathrm{CA}$ & White Mt. Peak (15') & 1962 & 14,246 \\
\hline North Palisade & $\mathrm{CA}$ & North Palisade & 1982 & 14,242 \\
\hline Mt. Cameron & $\mathrm{CO}$ & Alma & 1970 & 14,238 \\
\hline Mt. Shavano & $\mathrm{CO}$ & Maysville & 1983 & 14,229 \\
\hline Mt. Belford & $\mathrm{CO}$ & Mt. Harvard & 1982 & 14,197 \\
\hline Mt. Princeton & $\mathrm{CO}$ & Mt. Antero & 1982 & $14 \overline{197}$ \\
\hline Crestone Needle & $\mathrm{CO}$ & Crestone Peak & 1967 & 14,197 \\
\hline Mt. Yale & $\mathrm{CO}$ & Mt. Harvard & 1982 & 14,196 \\
\hline Mt. Bross & $\mathrm{CO}$ & Alma & 1970 & $\overline{14,172}$ \\
\hline Kit Carson Mt. & $\mathrm{CO}$ & Crestone Peak & 1967 & 14,165 \\
\hline Mt. Wrangell & $\mathrm{AK}$ & Gulkana A-1 & 1959 & 14,163 \\
\hline Mt. Shasta & $\mathrm{CA}$ & Shasta & 1986 & 14,162 \\
\hline El Diente Peak & $\mathrm{CO}$ & Dolores Peak & 1953 & 14,159 \\
\hline Point Success & WA & Mt. Rainier West & 1971 & 14,158 \\
\hline Maroon Peak & $\mathrm{CO}$ & Maroon Bells & 1960 & 14,156 \\
\hline Taberguache Mt. & $\mathrm{CO}$ & St. Elmo & 1982 & 14,155 \\
\hline Mt. Oxford & $\mathrm{CO}$ & Mt. Harvard & 1982 & 14,153 \\
\hline Mt. Sill & $\mathrm{CA}$ & North Palisade & 1982 & 14,153 \\
\hline Mt. Sneffels & $\mathrm{CO}$ & Mt. Sneffels & 1967 & 14,150 \\
\hline Mt. Democrat & $\mathrm{CO}$ & Climax & 1970 & 14,148 \\
\hline Capitol Peak & $\mathrm{CO}$ & Capitol Peak & 1960 & 14,130 \\
\hline Liberty Cap & WA & Mt. Rainier West & 1971 & 14,112 \\
\hline Pikes Peak & $\mathrm{CO}$ & Pikes Peak & 1951 & 14,110 \\
\hline Snowmass Mt. & $\mathrm{CO}$ & Snowmass Mt. & 1960 & 14,092 \\
\hline Mt. Russell & $\mathrm{CA}$ & Mt. Whitney & 1985 & 14,088 \\
\hline Mt. Eolus & $\mathrm{CO}$ & Mt. Whitney & 1985 & 14,083 \\
\hline Windom Peak & $\mathrm{CO}$ & Columbine Pass & 1973 & 14,082 \\
\hline Mt. Columbia & $\mathrm{CO}$ & Mt. Harvard & 1982 & 14,073 \\
\hline Mt. Augusta & AK & Mt. St. Elias & 1985 & 14,070 \\
\hline Missouri Mt. & $\mathrm{CO}$ & Winfield & 1982 & 14,067 \\
\hline Humboldt Peak & $\mathrm{CO}$ & Crestone Peak & 1967 & 14,064 \\
\hline Mt. Bierstadt & $\mathrm{CO}$ & Mt. Evans & 1957 & 14,060 \\
\hline Sunlight Peak & $\mathrm{CO}$ & Storm King Peak & 1964 & 14,059 \\
\hline Split Mt. & $\mathrm{CA}$ & Split Mt. & 1984 & 14,058 \\
\hline
\end{tabular}




\begin{tabular}{lllll}
\hline \multicolumn{1}{c}{ Summit } & State & Quadrangle & Date & $\begin{array}{c}\text { Elevation } \\
\text { feet }\end{array}$ \\
\hline Handies Peak & CO & Handies Peak & 1955 & 14,048 \\
\hline Culebra Peak & CO & Culebra Peak & 1955 & 14,047 \\
\hline Mt. Lindsey & CO & Blanca Peak & 1967 & 14,042 \\
\hline Ellingwood Point & CO & Blanca Peak & 1967 & 14,042 \\
\hline Little Bear Peak & CO & Blanca Peak & 1967 & 14,037 \\
\hline Mt. Sherman & CO & Mt. Sherman & 1961 & 14,036 \\
\hline Redcloud Peak & CO & Redcloud Peak & 1964 & 14,034 \\
\hline Mt. Langley & CA & Mt. Langley & 1982 & 14,026 \\
\hline Conundrum Peak & CO & Hayden Peak & 1960 & 14,022 \\
\hline Mt. Tyndall & CA & Mt. Williamson & 1984 & 14,019 \\
\hline Pyramid Peak & CO & Maroon Bells & 1960 & 14,018 \\
\hline Wilson Peak & CO & Mt. Wilson & 1953 & 14,017 \\
\hline Wetterhorn Peak & CO & Wetterhorn Peak & 1963 & 14,015 \\
\hline North Maroon Peak & CO & Maroon Bells & 1960 & 14,014 \\
\hline San Luis Peak & CO & San Luis Peak & 1986 & 14,014 \\
\hline Mt. Muir & CA & Mt. Whitney & 1985 & 14,012 \\
\hline Middle Palisade & CA & Split Mt. & 1984 & 14,012 \\
\hline Mt. of the & CO & Mt. of the Holy Cross & 1970 & 14,005 \\
\hline Holy Cross & & & & 1982 \\
\hline Huron Peak & CO & Winfield & 1982 & 14,003 \\
\hline Thunderbolt Peak & CA & North Palisade & 1964 & 14,003 \\
\hline Sunshine Peak & CO & Redcloud Peak & & 14,001 \\
\hline 1 Nationat Geodic & Sur & & & \\
\hline
\end{tabular}

1 National Geodetic Survey.

2USGS 7.5-minute quadrangle map in the conterminous United States unless otherwise indicated and 15-minute maps in Alaska unless otherwise indicated.

\section{Elevations of Selected Summits East of the Rocky Mountains}

\begin{tabular}{llllll}
\hline \multicolumn{1}{c}{ Summit } & State & County & Quadrangle & \multicolumn{2}{c}{$\begin{array}{c}\text { Date } \\
\text { Elevation } \\
\text { feet }\end{array}$} \\
\hline White Mountains, Presidential Range: & $\mathrm{NH}$ & Coos & Mt. Washington & 1982 & 6,288 \\
\hline Mt. Washington & $\mathrm{NH}$ & Coos & Mt. Washington & 1982 & 5,774 \\
\hline Mt. Adams & $\mathrm{NH}$ & Coos & Mt. Washington & 1982 & 5,712 \\
\hline Mt. Jefferson & $\mathrm{NH}$ & Coos & Mt. Washington & 1982 & 5,584 \\
\hline Mt. Sam Adams & $\mathrm{NH}$ & Coos & Mt. Washington & 1982 & 5,532 \\
\hline Mt. Clay & $\mathrm{NH}$ & Coos & Mt. Washington & 1982 & 5,492 \\
\hline Boott Spur & $\mathrm{NH}$ & Coos & Mt. Washington & 1982 & 5,394 \\
\hline Mt. Quincy Adams & $\mathrm{NH}$ & Coos & Mt. Washington & 1982 & 5,374 \\
\hline Mt. Monroe & $\mathrm{NH}$ & Coos & Mt. Washington & 1982 & 5,367 \\
\hline Mt. Madison & $\mathrm{NH}$ & Grafton & Franconia & 1967 & 5,249 \\
\hline Mt. Lafayette & $\mathrm{NH}$ & Grafton & Franconia & 1967 & 5,089 \\
\hline Mt. Lincoln & $\mathrm{NH}$ & Coos & Crawford Notch & 1946 & 5,004 \\
\hline Mt. Franklin & $\mathrm{NH}$ & Grafton & South Twin Mt. & 1967 & 4,902 \\
\hline South Twin Mt. & & & &
\end{tabular}


Summit State County Quadrangle $^{3}$ Date Elevation

Adirondack Mountains, Mt. Marcy area:

\begin{tabular}{llllll}
\hline Mt. Marcy & NY & Essex & Mt. Marcy & 1979 & 5,344 \\
\hline Algonquin Peak & NY & Essex & Mt. Marcy & 1979 & 5,115 \\
\hline Mt. Haystack & NY & Essex & Mt. Marcy & 1979 & 4,961 \\
\hline Mt. Skylight & NY & Essex & Mt. Marcy & 1979 & 4,925 \\
\hline Whiteface Mt. & NY & Essex & Lake Placid & 1978 & 4,865 \\
\hline Iroquois Peak & NY & Essex & Mt. Marcy & 1979 & 4,849 \\
\hline Boundary Peak & NY & Essex & Mt. Marcy & 1979 & 4,829 \\
\hline Gray Peak & NY & Essex & Mt. Marcy & 1979 & 4,826 \\
\hline Basin Mt. & NY & Essex & Mt. Marcy & 1979 & 4,826 \\
\hline Dix Mt. & NY & Essex & Mt. Marcy & 1979 & 4,822 \\
\hline Little Marcy & NY & Essex & Mt. Marcy & 1979 & 4,744 \\
\hline Gothics & NY & Essex & Keene Valley & 1979 & 4,734 \\
\hline
\end{tabular}

\begin{tabular}{llllll}
\multicolumn{1}{c}{ Summit } & State & County & Quadrangle $^{3}$ & \multicolumn{1}{c}{$\begin{array}{c}\text { Date } \\
\text { Elevation } \\
\text { feet }\end{array}$} \\
\hline Catskill Mountains: & & & & & \\
\hline Slide Mt. & NY & Ulster & Peekamoose & 1969 & 4,180 \\
\hline Hunter Mt. & NY & Greene & Hunter & 1945 & 4,040 \\
\hline Black Dome & NY & Greene & Freehold & 1943 & 3,980 \\
\hline Blackhead & NY & Greene & Freehold & 1943 & 3,940 \\
\hline Thomas Cole Mt. & NY & Greene & Hensonville & 1945 & 3,940 \\
\hline West Kill Mt. & NY & Greene & Lexington & 1960 & 3,880 \\
\hline Graham Mt. & NY & Ulster & Seager & 1945 & 3,868 \\
\hline Cornell Mt. & NY & Ulster & Phoenicia & 1950 & 3,860 \\
\hline Doubletop Mt. & NY & Ulster & Seager & 1945 & 3,860 \\
\hline Table Mt. & NY & Ulster & Peekamoose & 1969 & 3,847 \\
\hline Peekamoose Mt. & NY & Ulster & Peekamoose & 1969 & 3,843 \\
\hline Plateau Mt. & NY & Greene & Hunter & 1945 & 3,840 \\
\hline
\end{tabular}

\section{$\begin{array}{llll}\text { Summit } & \text { State } & \text { Quadrangle } & \\ & \text { Date Elevation }\end{array}$}

feet

Blue Ridge and Great Smoky Mountains:

\begin{tabular}{llllll}
\hline Mt. Mitchell & NC & Yancey & Mt. Mitchell & 1946 & 6,684 \\
\hline Mt. Craig & NC & Yancey & Mt. Mitchell & 1946 & 6,647 \\
\hline Clingmans Dome & NC-TN & Swain-Sevier & Clingmans Dome & 1964 & 6,643 \\
\hline Mt. Guyot & NC-TN & Haywood-Sevier & Mt. Guyot & 1964 & 6,621 \\
\hline Balsam Cone & NC & Yancey & Mt. Mitchell & 1946 & 6,600 \\
\hline Cattail Peak & NC & Yancey & Mt. Mitchell & 1946 & 6,600 \\
\hline Mt. Le Conte & NC & Sevier & Mt. Le Conte & 1964 & 6,593 \\
\hline Big Tom & NC & Yancey & Mt. Mitchell & 1946 & 6,526 \\
\hline
\end{tabular}




\begin{tabular}{lcllrr}
\multicolumn{1}{c}{ Summit } & State & County & Quadrangle & \multicolumn{2}{c}{$\begin{array}{c}\text { Date } \\
\text { Elevation } \\
\text { feet }\end{array}$} \\
\hline Mt. Gibbes & NC & Yancey & Montreat & 1942 & 6,520 \\
\hline Potato Hill & NC & Yancey & Mt. Mitchell & 1946 & 6,440 \\
\hline Mt. Chapman & NC-TN & Swain-Sevier & Mt. Guyot & 1964 & 6,417 \\
\hline Old Black & NC-TN & $\begin{array}{l}\text { Haywood-Cocke- } \\
\text { Sevier }\end{array}$ & Mt. Guyot & 1964 & 6,370 \\
\hline Celo Knob & NC & Yancey & Celo & 1960 & 6,327 \\
\hline
\end{tabular}

\begin{tabular}{|c|c|c|c|c|c|}
\hline Summit & State & County & Quadrangle $^{3}$ & Date & $\begin{array}{c}\text { Elevation } \\
\text { feet }\end{array}$ \\
\hline \multicolumn{6}{|c|}{ Ozark-Ouachita Mountains: } \\
\hline Magazine Mt. & AR & Logan & Blue Mt. & 1966 & 2,753 \\
\hline Rich Mt. & AR & Polk & Rich Mt. & 1958 & 2,681 \\
\hline Poteau Mt. & AR & Sebastian & Cauthron & 1958 & 2,669 \\
\hline Black Fork Mt. & AR & Polk & Mountain Fork & 1958 & 2,586 \\
\hline Sugar Loaf Mt. & OK & Le Flore & Hartford & 1947 & 2,564 \\
\hline Buffalo Lookout & AR & Newton & Fallsville & 1967 & 2,561 \\
\hline Wilton Mt. & OK & Le Flore & Page & 1981 & 2,558 \\
\hline Lynn Mt. & OK & Le Flore & Lynn Mt. & 1981 & 2,480 \\
\hline Turner Ward Knob & AR & Newton & Fallsville & 1947 & 2,463 \\
\hline Kiamichi Mt. & OK & Le Flore & Muse & 1966 & 2,451 \\
\hline Winding Stair Mt. & $\mathrm{OK}$ & Le Flore & Big Cedar & 1981 & $2, \overline{451}$ \\
\hline Reeves Mt. & AR & Newton & Boxley & 1968 & 2,440 \\
\hline
\end{tabular}

\begin{tabular}{|c|c|c|c|c|c|}
\hline Summit & State & County & Quadrangle $^{3}$ & Date & $\begin{array}{c}\text { Elevation } \\
\text { feet }\end{array}$ \\
\hline \multicolumn{6}{|l|}{ Black Hills: } \\
\hline Harney Peak ${ }^{2}$ & SD & Pennington & Custer & 1957 & 7,242 \\
\hline Bear Mt. & $S D$ & Pennington & Berne & 1954 & 7,166 \\
\hline Crooks Tower & SD & Lawrence & Crooks Tower & 1956 & 7,137 \\
\hline Terry Peak & SD & Lawrence & Lead & 1961 & 7,064 \\
\hline Crows Nest Peak & SD & Pennington & Crows Nest Peak & 1956 & 7,048 \\
\hline White Tail Peak & SD & Pennington & Deerfield & 1956 & 6,962 \\
\hline Flag Mt. & SD & Pennington & Deerfield & 1956 & 6,937 \\
\hline Medicine Mt. & SD & Pennington & Medicine Mt. & 1956 & 6,878 \\
\hline Nipple Butte & SD & Pennington & Deerfield & 1956 & 6,810 \\
\hline Custer Peak & SD & Lawrence & Minnesota Ridge & 1956 & 6,804 \\
\hline Mt. Rushmore & SD & Pennington & Mt. Rushmore & 1954 & 5,725 \\
\hline Devils Tower & WY & Crook & Devils Tower & 1984 & 5,112 \\
\hline
\end{tabular}

${ }^{1}$ Highest summit east of the Mississippi River.

${ }^{2}$ Highest summit east of the Rocky Mts.

3USGS 7.5 -minute or 7.5-minute by 15 -minute quadrangle map unless otherwise indicated. 


\section{Geographic Centers}

Because there is no generally accepted definition of a geographic center and no completely satisfactory method of determining it, there may be as many geographic centers of a State or country as there are definitions of the term.

The geographic center of an area may be defined as the center of gravity of the surface, or that point on which the surface of an area would balance if it were a plane of uniform thickness.

Curvature of the Earth, large bodies of water, irregular surfaces, and other factors affect the determination of center of gravity.

In determining the centers of the States, islands adjacent to their coastlines and large bodies of water were excluded.

The geographic centers and positions listed below should be considered as approximations.

\section{State}

Alabama

Alaska

Arizona

Arkansas

California

Colorado

Connecticut

Delaware

District of Columbia

Florida

Georgia

Hawaii

Idaho

Illinois

Indiana

lowa

Kansas

Kentucky

Louisiana

Maine

Maryland

Massachusetts

Michigan

Minnesota

Mississippi

Missouri

Montana

Nebraska

Nevada

New Hampshire

New Jersey

New Mexico
County

Chilton

$63^{\circ} 50^{\prime} \mathrm{N} ., 1^{\circ} 00^{\prime} \mathrm{W}$.

Yavapai

Pulaski

Madera

Park

Hartford

Kent

Hernando

Twiggs

Hawaii

Custer

Logan

Boone

Story

Barton

Marion

Avoyelles

Piscataquis

Prince Georges

Worcester

Wexford

Crow Wing

Leake

Miller

Fergus

Custer

Lander

Belknap

Mercer

Torrance

\section{Locality}

12 miles southwest of Clanton

60 miles northwest of Mt. McKinley

55 miles east-southeast of Prescott

12 miles northwest of Little Rock

38 miles east of Madera

30 miles northwest of Pikes Peak

At East Berlin

11 miles south of Dover

Near Fourth and L Streets N.W.

12 miles north-northwest of Brooksville

18 miles southeast of Macon

$20^{\circ} 15^{\prime}$ N., $156^{\circ} 20^{\prime} W$., off Maui Island

At Custer, southwest of Challis

28 miles northeast of Springfield

14 miles north-northwest of Indianapolis

5 miles northeast of Ames

15 miles northeast of Great Bend

3 miles north-northwest of Lebanon

3 miles southeast of Marksville

18 miles north of Dover

4 1/2 miles northwest of Davidsonville

North part of City of Worcester

5 miles north-northwest of Cadillac

10 miles southwest of Brainerd

9 miles west-northwest of Carthage

20 miles southwest of Jefferson City

11 miles west of Lewistown

10 miles northwest of Broken Bow

26 miles southeast of Austin

3 miles east of Ashland

5 miles southeast of Trenton

12 miles south-southwest of Willard 


\begin{tabular}{lll}
$\begin{array}{l}\text { State } \\
\text { New York }\end{array}$ & Madison & \multicolumn{1}{c}{ County } \\
\hline North Carolina & Chatham & $\begin{array}{c}12 \text { miles south of Oneida and 26 miles } \\
\text { southwest of Utica }\end{array}$ \\
\hline North Dakota & Sheridan & 10 miles northwest of Sanford \\
\hline Ohio & Delaware & 5 miles southwest of McClusky \\
\hline Oklahoma & Oklahoma & 25 miles north-northeast of Columbus \\
\hline Oregon & Crook & 8 miles north of Oklahoma City \\
\hline Pennsylvania & Centre & 25 miles south-southeast of Prineville \\
\hline Rhode Island & Kent & $21 / 2$ miles southwest of Bellefonte \\
\hline South Carolina & Richland & 1 mile south-southwest of Crompton \\
\hline South Dakota & Hughes & 13 miles southeast of Columbia \\
\hline Tennessee & Rutherford & 8 miles northeast of Pierre \\
\hline Texas & McCulloch & 5 miles northeast of Murfreesboro \\
\hline Utah & Sanpete & 15 miles northeast of Brady \\
\hline Vermont & Washington & 3 miles north of Manti \\
\hline Virginia & Buckingham & 3 miles east of Roxbury \\
\hline Washington & Chelan & 5 miles southwest of Buckingham \\
\hline West Virginia & Braxton & 10 miles west-southwest of Wenatchee \\
\hline Wisconsin & Wood & 4 miles east of Sutton \\
\hline Wyoming & Fremont & 9 miles southeast of Marshfield \\
\hline
\end{tabular}

\begin{tabular}{llll}
$\begin{array}{l}\text { Geographic } \\
\text { area }\end{array}$ & \multicolumn{1}{c}{ Locality } & $\begin{array}{c}\text { Latitude } \\
\text { (N) }\end{array}$ & $\begin{array}{c}\text { Longitude } \\
\text { (W) }\end{array}$ \\
\hline $\begin{array}{l}\text { Conterminous } \\
\text { United States } \\
\text { (48 States) }\end{array}$ & Near Lebanon, Smith County, Kansas & $39^{\circ} 50^{\prime}$ & $98^{\circ} 35^{\prime}$ \\
\hline $\begin{array}{l}\text { Continental United } \\
\text { States (49 States) }\end{array}$ & Near Castle Rock, Butte County, South Dakota & $44^{\circ} 59^{\prime}$ & $103^{\circ} 38^{\prime}$ \\
\hline $\begin{array}{l}\text { The United States } \\
\text { (50 States) }\end{array}$ & West of Castle Rock, Butte County, South Dakota & $44^{\circ} 58^{\prime}$ & $103^{\circ} 46^{\prime}$ \\
\hline $\begin{array}{l}\text { North American } \\
\text { Continent }\end{array}$ & 6 miles west of Balta, Pierce County, North Dakota $48^{\circ} 10^{\prime}$ & $100^{\circ} 10^{\prime}$ \\
\hline
\end{tabular}

No marked or monumented point has been established by any government agency as the geographic center of either the 50 States, the conterminous United States, or the North American continent. However, a monument was erected in Lebanon, Kansas, by a group of citizens who hired engineers to determine the position of the "geographic center" of the United States.

Sometimes confused with the geographic center of the United States is the triangulation station "Meades Ranch," located at latitude 39¹3'26.686" North and longitude $98^{\circ} 32$ '30.506" West, about 12 miles north of Lucas, Kansas. This was the primary reference point for the North American Datum of 1927, the standard on which all the latitude and longitude lines for North America were based. This datum is being replaced by the North American Datum of 1983 , which is not hinged to a single point of reference. 
Distances From Extreme Points to Geographic Centers

\begin{tabular}{|c|c|c|c|c|c|c|}
\hline Extreme point & $\begin{array}{l}\text { Coor } \\
\text { Latitude } \\
\text { (N) }\end{array}$ & $\begin{array}{l}\text { dinates } \\
\text { Longitude } \\
\text { (W) }\end{array}$ & $\begin{array}{l}\text { Direction } \\
\text { from geo- } \\
\text { graphic } \\
\text { centers } \\
\end{array}$ & $\begin{array}{l}50 \text { States* } \\
\text { Statute } \\
\text { miles }\end{array}$ & $\begin{array}{l}49 \text { States * * } \\
\text { Statute } \\
\text { miles }\end{array}$ & $\begin{array}{l}48 \text { States** } \\
\text { Statute } \\
\text { miles } \\
\end{array}$ \\
\hline Point Barrow, AK & $71^{\circ} 23^{\prime}$ & $156^{\circ} 29^{\prime}$ & Northwest & 2,507 & 2,509 & 2,940 \\
\hline $\begin{array}{l}\text { Pochnoi Point, } \\
\text { Semisopochnoi, } \\
\text { AK }\end{array}$ & $51^{\circ} 57^{\prime}$ & $179^{\circ} 52^{\prime}(E)$ & Northwest & 3,372 & 3,377 & 3,802 \\
\hline $\begin{array}{l}\text { West Point of } \\
\text { Amatignak Island, } \\
\text { AK }\end{array}$ & $51^{\circ} 17^{\prime}$ & $179^{\circ} 09^{\prime}$ & Northwest & 3,352 & 3,357 & 3,780 \\
\hline $\begin{array}{l}\text { Cape Wrangell, } \\
\text { Attu Island, AK }\end{array}$ & $52^{\circ} 55^{\prime}$ & $172^{\circ} 27^{\prime}(\mathrm{E})$ & Northwest & 3,625 & 3,630 & 4,061 \\
\hline $\begin{array}{l}\text { Lake of the Woods } \\
\text { Projection, MN }\end{array}$ & $49^{\circ} 23^{\prime}$ & $95^{\circ} 09^{\prime}$ & Northeast & 507 & 501 & 1682 \\
\hline $\begin{array}{l}\text { North end,(N-S } \\
\text { boundary) ME- } \\
\text { New Brunswick, } \\
\text { Canada } \\
\end{array}$ & $47^{\circ} 04^{\prime}$ & $67^{\circ} 47^{\prime}$ & Northeast & 1,719 & 1,713 & 1,613 \\
\hline $\begin{array}{l}\text { West Quoddy } \\
\text { Head, ME }\end{array}$ & $44^{\circ} 49^{\prime}$ & $66^{\circ} 57^{\prime}$ & East & 1,788 & 1,782 & 21,643 \\
\hline Key West, FL & $24^{\circ} 32^{\prime}$ & $81^{\circ} 48^{\prime}$ & Southeast & 1,873 & 1,869 & 1,439 \\
\hline Cape Sable, FL & $25^{\circ} 07^{\prime}$ & $81^{\circ} 05^{\prime}$ & Southeast & 1,867 & 1,863 & 1,437 \\
\hline $\begin{array}{l}\text { Log Point, Elliot } \\
\text { Key, FL }\end{array}$ & $25^{\circ} 29^{\prime}$ & $80^{\circ} 11^{\prime}$ & Southeast & 1,882 & 1,877 & 1,455 \\
\hline $\begin{array}{l}\text { East Point, St. } \\
\text { Croix, VI }\end{array}$ & $17^{\circ} 45^{\prime}$ & $64^{\circ} 34^{\prime}$ & Southeast & 2,938 & 2,932 & 2,540 \\
\hline $\begin{array}{l}\text { Extreme south } \\
\text { point of TX }\end{array}$ & $25^{\circ} 50^{\prime}$ & $97^{\circ} 24^{\prime}$ & South & 1,370 & 1,369 & 971 \\
\hline $\begin{array}{l}\text { Steps Point, } \\
\text { America Samoa }\end{array}$ & $14^{\circ} 23^{\prime}(\mathrm{S})$ & $170^{\circ} 46^{\prime}$ & Southwest & 5,859 & 5,866 & 5,954 \\
\hline Orote Point, Guam & $13^{\circ} 27^{\prime}$ & $144^{\circ} 37^{\prime}(\mathrm{E})$ & Southwest & 6,579 & 6,584 & 6,974 \\
\hline Kure Island, $\mathrm{HI}$ & $28^{\circ} 25^{\prime}$ & $178^{\circ} 20^{\prime}$ & Southwest & 4,142 & 4,148 & 4,486 \\
\hline Ka Lae, HI & $18^{\circ} 55^{\prime}$ & $155^{\circ} 41^{\prime}$ & Southwest & 3,463 & 3,470 & 3,664 \\
\hline $\begin{array}{l}\text { Cape Mendocino, } \\
\text { CA }\end{array}$ & $40^{\circ} 26^{\prime}$ & $124^{\circ} 24^{\prime}$ & West & 1,091 & 1,098 & 1,361 \\
\hline Cape Alava, WA & $48^{\circ} 10^{\prime}$ & $124^{\circ} 44^{\prime}$ & West & 1,018 & 1,024 & 1,414 \\
\hline
\end{tabular}

*West of Castle Rock, Butte County, South Dakota. $44^{\circ} 58^{\prime} \mathrm{N}-103^{\circ} 46^{\prime} \mathrm{W}$

* *Near Castle Rock, Butte County, South Dakota. 44⒌

** *Near Lebanon, Smith County, Kansas. 39 $50^{\prime} \mathrm{N}-98^{\circ} 35^{\prime} \mathrm{W}$

1 General direction to Lake of the Woods Projection is north.

${ }^{2}$ General direction to West Quoddy Head is northeast. 


\section{Extreme Distances (computed to mean sea level)}

\begin{tabular}{|c|c|c|}
\hline From & To & $\begin{array}{c}\text { Statute } \\
\text { miles }\end{array}$ \\
\hline West Quoddy Head, ME, along the parallel & Pacific Ocean & 2,742 \\
\hline West Quoddy Head, ME & Cape Wrangell, AK & 4,831 \\
\hline West Quoddy Head, ME & Kure Island, Hawaii & 5,797 \\
\hline West Quoddy Head, ME & $\begin{array}{l}\text { Point Arena, CA (farthest points, } 48 \\
\text { States) }\end{array}$ & 2,892 \\
\hline Log Point, Elliot Key, FL & $\begin{array}{l}\text { Kure Island, Hawaii (farthest points, } 50 \\
\text { States) }\end{array}$ & $\begin{array}{l}0 \\
5,859 \\
\end{array}$ \\
\hline Log Point, Elliot Key, FL & Cape Wrangell, AK & 5,505 \\
\hline Soldier Key, FL & Kure Island, Hawaii & 5,859 \\
\hline Soldier Key, FL & Cape Wrangell, AK & 5,503 \\
\hline Rivera Beach, FL & Kure Island, Hawaii & 5,823 \\
\hline East Point, St. Croix, VI & Cape Wrangell, AK & 6,501 \\
\hline East Point, St. Croix, VI & $\begin{array}{l}\text { Orote Point, Guam (longest distance } \\
\text { including outlying areas) }\end{array}$ & 9,514 \\
\hline $\begin{array}{l}\text { Approximately } 10 \text { miles south of } \\
\text { Brunswick, GA }\end{array}$ & $\begin{array}{l}\text { Approximately } 12 \text { miles south of San } \\
\text { Diego, CA (shortest coast-to-coast } \\
\text { distance, } 48 \text { States) }\end{array}$ & 2,089 \\
\hline $\begin{array}{l}\text { North end of ME-New Brunswick, Canada } \\
\text { boundary }\end{array}$ & Orote Point, Guam & 7,747 \\
\hline Point Barrow, AK & $\begin{array}{l}\text { Steps Point, Tutuila Island, American } \\
\text { Samoa }\end{array}$ & 5,971 \\
\hline Southern Point of TX, due north & 49th parallel & 1,602 \\
\hline West point of Amatignak Island, AK & $\begin{array}{l}\text { Pochnoi Point, Semisopochnoi Island, } \\
\text { AK (distance between the most easter- } \\
\text { ly and the most westerly points in the } \\
\text { United States with reference to the } \\
180^{\circ} \text { meridian) }\end{array}$ &  \\
\hline
\end{tabular}

\section{Lengths of United States Boundaries}

\begin{tabular}{lr}
\hline Northern boundary of conterminous United States & $\begin{array}{r}\text { Statute } \\
\text { miles }\end{array}$ \\
\hline Canada-Alaska boundary & 3,987 \\
\hline Southern boundary of conterminous United States & 1,538 \\
\hline
\end{tabular}


